\title{
Real Time Measurement and Processing of Pupillary Light Reflex for Early Detection of Disease
}

\author{
Ippei Torii*, Takahito Niwa \\ Aichi Institute of Technology, Dept. of Information Science, 1247 Yachigusa, Yakusa-cho, Toyota, Aichi, Japan. \\ * Corresponding author. Tel.: 81-565-48-8121; email: mac[aitech.ac.jp \\ Manuscript submitted January 10, 2019; accepted March 8, 2019. \\ doi: $10.17706 /$ jcp.14.3.161-169
}

\begin{abstract}
Recently, pupillary measurements have begun to be considered effective in the diagnosis of various physical conditions. Apart from optic neuropathy and retinal disorders, which are ocular diseases, diversions can be expected in the diagnosis of autonomic disturbance due to sympathetic and parasympathetic nerve disorders, cranial nerve disorders, cerebral infarction, depression, and diabetes. In this study, we have developed a real-time pupil diameter measuring system that is inexpensive and does not require a complicated device. When strong light is projected onto the eyeball, this system can measure the reaction time until the start of miosis, the time to achieve maximal miosis, and the difference in reaction speed of the left and right eyes. With this system, the status of a disease can be judged based on the distance from the threshold value.
\end{abstract}

Key words: Real time measurement, eye movement, early detection of disease, image processing.

\section{Introduction}

There are approximately one hundred million photoreceptor cells in the retina of human eyes. Light that shines on those cells is converted to nerve signals, which transmit the information to the brain, resulting in the visual recognition of objects. When light is radiated onto the eye, the pupillary light reflex is triggered, causing the pupillary diameter to decrease with a time delay of less than $1 \mathrm{sec}$. A larger area of radiated light causes a stronger reflex reaction. Pupil observation is a basic technique in clinical neurology [1]. It is of particular significance in patients with impaired consciousness [2], as it can become the basis on which causal factors and locations of lesions are diagnosed; moreover, it can become the basis on which severity, prognosis, and indications for surgical intervention are judged. Pupillary abnormalities are induced due to compression on the oculomotor nerve, as well as brain stem ischemia; recovery is possible with early treatment intervention [3]. Pupillary diameter and the shape, status, and speed of pupillary light reflex are observed; however, in textbooks, pupillary light reflex is classified only as brisk or prompt (fast), sluggish (slow, delayed, blunted), and non-reactive or absent (disappeared, none). As such, a subjective decision is made by the observer. Hence, inter-observer differences are large and a lack of objectivity has been noted [4], [5].

Pupillary light reflex varies according to pupillary diameter and light intensity. Because it adjusts the amount of light reaching the retina, the pupillary light reflex is very important in neurological evaluations. In addition to diagnosing optic nerve disorders and retinal disorders that are induced by abnormalities in this reflex, the pupillary light reflex can be applied in the diagnosis of autonomic disturbances due to sympathetic 
and parasympathetic nerve disorders, cranial nerve disorders, cerebral infarction, diabetes, depression, and various other factors. Moreover, the pupil reacts to over stimulation from bright light, such as in during operation of visual display terminals (smartphones and computer displays), causing fatigue of the iris sphincter muscles. The possibility of this inducing asthenopia, dry eye, and other conditions has been noted.

In the field of neuro-ophthalmology in Japan, the pupillary light reflex measuring device by Hamamatsu Photonics Inc., Iriscorder Dual C-10641, and Newopto ET-200 etc., are the primary devices used to determine the condition of the pupil. These devices record still images of the state of the pupil before and after exposure to light.

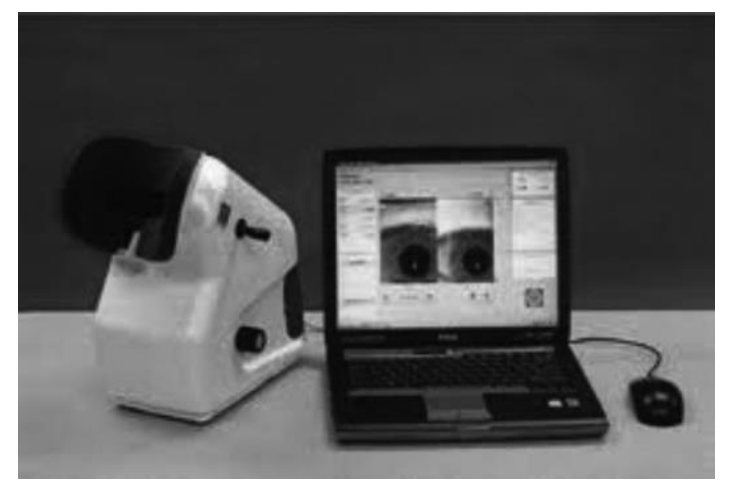

Fig. 1. An example of a conventional measuring instrument (Newopto ET - 200).

Rather than providing still images of the pupil, we have focused on the speed of the pupillary light reflex and amount of change in the pupillary region. We have developed a real-time measurement system that may enable early discovery of diseases, which naturally would prevent their aggravation, and possibly avoid the initial onset.

Studies thus far have focused on the research and development of communication tools based on the amount of change in the pixel count of the eye for children with disability, patients with amyotrophic lateral sclerosis, and the elderly, among others. Studies have also focused on communication tools based on steady gaze and viewing direction for individuals with difficulty in verbal or written communication. These tools are highly regarded in special schools across Japan, as well as in foreign countries. [Communication systems and blink detection methods (Patent No. 5871290)].

\section{Purpose of Study}

This study is performed to determine the amount of change in papillary pixel count based on "image processing by image retention," which is used in blink detection. Then, the difference between healthy volunteers and diseased patients is compared. Objective indicators (threshold) are then be established to elucidate the risk of disease. We also study the association of abnormal paralysis of the optic nerves in cerebral infarction and schizophrenic eye, with abnormal pupillary movement with progression and eyeball movement during early stages of amyotrophic lateral sclerosis.

There are many types of disease assessments, which vary according to the institution; there is also a wide range of confirmation items. As such, the diagnosis is currently entrusted to the subjective judgment of an experienced doctor. We aim to establish an assessment tool enabling accurate discrimination, whereby anyone could obtain the same quantitative judgement results for the early detection of disease and implementation of appropriate actions.

Typically, fundoscopy is performed after mydriasis. Regarding eye drops only, the effect of the medication diminishes within 4-5 hours. In this study, measurements could be performed by simply 
observing pupillary movement in both eyes for approximately $15 \mathrm{sec}$. Thus, measurements could be performed with adequate precision in a short time.

The purpose of this study is to enable the detection and measurement of the pupil, regardless of the color of the iris, by simply using a cheap device where the color of the iris is not affected when infrared radiation LED is used. Rather than using still images of the pupillary area that are taken with conventional approaches, the amount of change can be measured in real-time. This study of the amount of change in ocular and pupillary pixel count may become a test that is indispensable for the early discovery and follow-up of brain disorders (particularly depression), as well as to determine their correlations with ocular disorders and diabetes; it may become similarly important in the prevention, management, and follow-up of hypertension, myocardial infarction, and other diseases.

\section{Pupillary Light Reflex}

In the pupillary reaction, the retina is stimulated by changes in light intensity. The pupil constricts or dilates reflexively by the near reaction, which occurs when pupillary light reflex and focus adjustment are performed; the closed eyelid reaction, which occurs when the eyelids are tightly closed, or as a result of changes in trigeminal nerve stimulation, electrical stimulation, and vestibular nerve stimulation, among others; and reflexive reaction, which occurs in mydriasis due to mental variations. Moreover, the amount of light entering the eye changes rapidly, causing the pupil to dilate and constrict; this reaction is known as the pupillary light reflex. In the pupillary reaction, adjustment involves joint movement in both eyes, which is similar to that observed in convergence. When strong light rapidly enters one eye, miosis occurs both in that eye and in the fellow eye; this is known as the indirect response. This type of reflex movement is involuntary.

Because the pupillary light reflex is a type of reflex movement, there is a latent period prior to its occurrence. The duration of that period differs based on the intensity of light stimulation. When this is markedly decreased, it lasts for at least $0.5 \mathrm{sec}$. However, when the intensity of light stimulation is strong, it lasts for a shorter period, 0.2-0.3 sec. Compared with other reflex movements, this is a modest change.

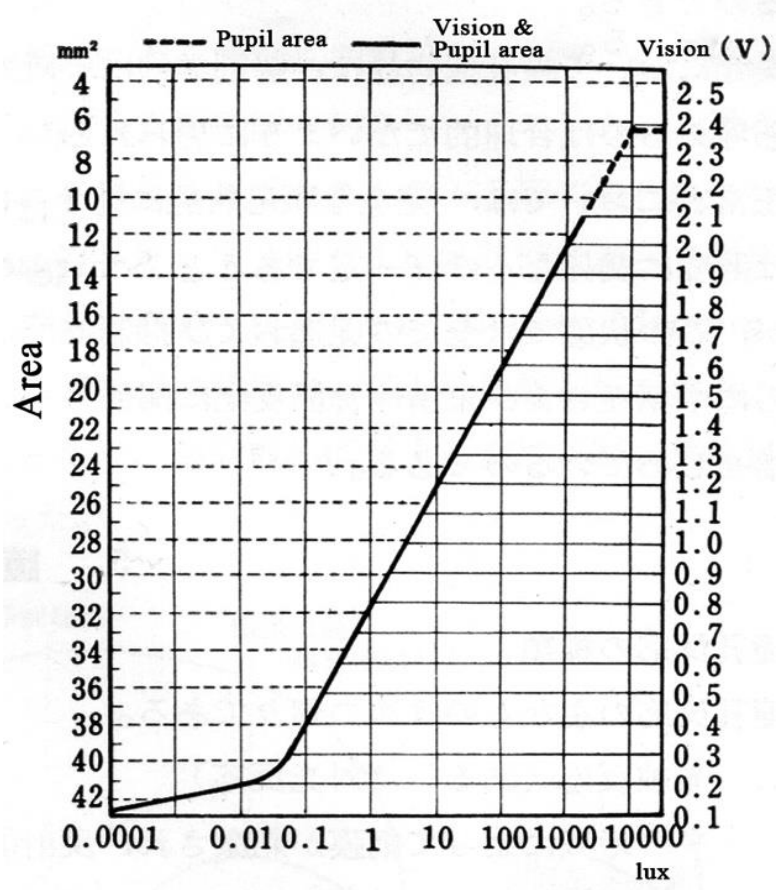

Fig. 2. Relationship between brightness of light source and pupil contraction speed. 
The time until peak miosis after light stimulation is known as the reflex time. This duration is typically approximately $1 \mathrm{sec}$, and is approximately identical regardless of light intensity and color. When the pupillary light reflex is analyzed in real time, the first $0.4 \mathrm{sec}$ comprises gradual constriction time, while the remaining constriction is fast (Fig. 2).

Acquisition of pupillary reaction speed, miosis, dilation speed, and indirect reaction from the characteristics of these pupillary light reflexes is linked to the development of a device that can easily measure physical disorders.

\section{Structure of the System}

This study develops a tool by which ocular and pupillary movements in multiple diseases, including cerebral infarction, can be measured, in order to improve ocular movement measuring technology. The first stage of the study is performed as outlined below.

1. We develop an ocular and pupillary movement measuring device (special goggles) using a near-infrared camera and Raspberry Pi compact computer.

2. We develop an LED emission pattern in the googles using a Windows laptop PC, and develop a data processing program of the images sent from the near-infrared camera.

3. We extract the pixels showing the pupillary area of the subject's eye, based on the acquired images.

4. We obtain the frame image (100 fps) using the after-image.

5. We develop programs that can perform pupillary reaction measurements in the pupillary light reflex of the subject.

\subsection{Development of the Pupil Movement Measuring Device}

The amount of change in pupillary pixel count is determined based on existing research and development, and disease status is determined from the speed of the pupillary light reflex. The pupil is measured using goggles; the LED mounted inside the device is flashed and the reaction is measured. Fig. 3 shows the ocular movement measurement device that was developed.

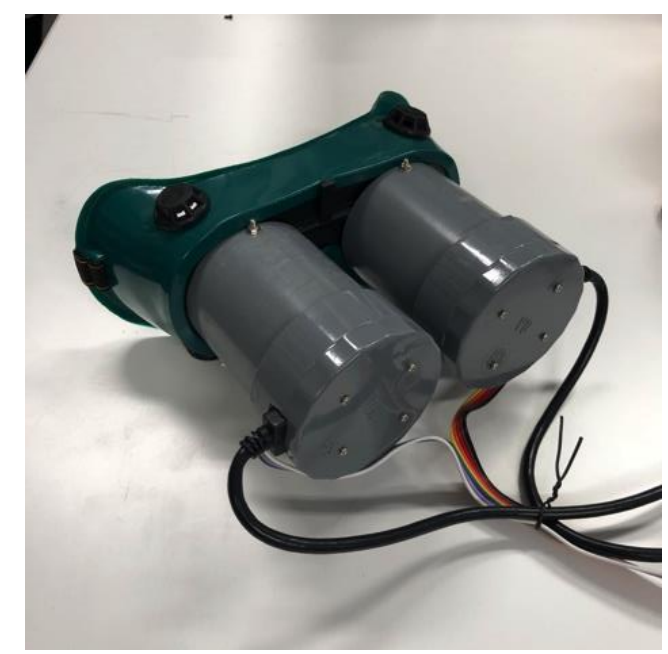

Fig. 3. Appearance of goggles.

Near-infrared cameras (GAZO Inc.) were installed inside the left and right goggles. In addition, Raspberry $\mathrm{Pi}$, a compact single board computer with a built-in ARM processor, was installed in the device. In addition, operations were performed to synchronize the ocular movement measuring program and the timing when light-emitting diodes emit light. 


\subsection{Pupil Detection Method Using the After-Image}

With regard to the detection of the iris and pupil, many studies thus far use Hough conversion and extraction methods, which utilize least median and Eye models for the detection of round and elliptical shapes. However, for real-time detection, data processing is a burden; therefore, measurement of the mean $100 \mathrm{fps}$, which is required in this study, cannot be performed.

Hence, we improved the detection method using the after-image for detecting blink and gaze that we have developed to date [6], [7]. Compared with other pupil-iris areas, the pupil itself does not reflect light; therefore, when light passes through an infrared camera, there is a difference in brightness, regardless of the color of the eyes. The site of the pupil is then detected following thresholding. Furthermore, we have developed a pupil detection method, where the color of all pixels obtained in each frame is identified as black or another color. Then, by recalling the sites of the black pixels, the pupil can be measured in real-time (Fig. 4).

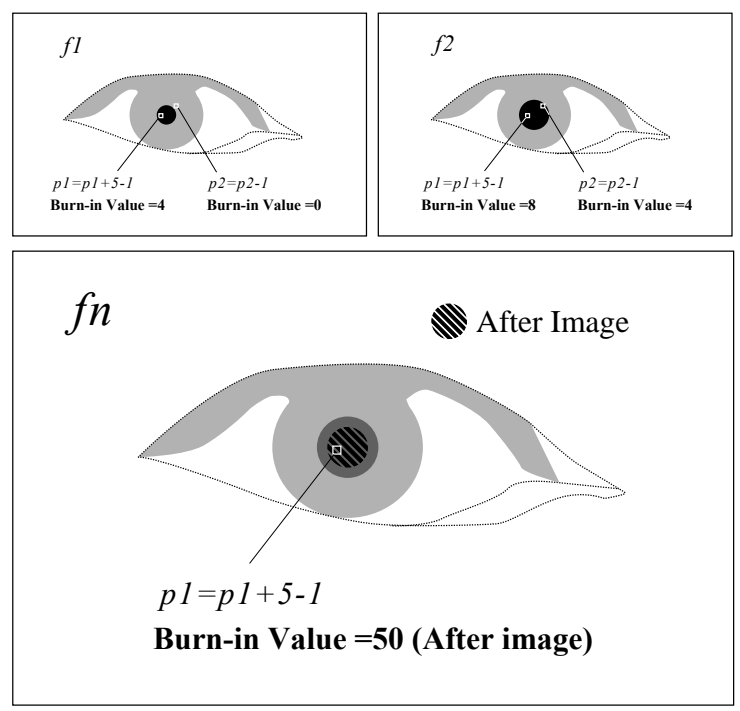

Fig. 4. Measurement technique for acquiring pupil movement: pupil detection method using afterimage.

With respect to instances where the pupil is undetected due to blinking, these were resolved by setting the threshold of change regarding the amount of change in pixel count, and by automatically excluding frames exceeding the threshold from the results of the total measurement.

\subsection{Analysis Methods}

With the system that was developed by this study, pupillary light reflex movement (both eyes) and alternating pupillary light reflex movement (one eye) are measured. The pupillary light reflex test is a method in which light is shone on to the fovea located behind the pupil of one eye to confirm the direct reaction, which comprises miosis of the eye exposed to light, and the indirect reaction, which comprises miosis of the eye not exposed to light. The status of disease is then judged based on the speed of the direct reaction (fast or slow), and the extent of miosis (adequate or inadequate). Here, two time periods, namely, the time to the start of miosis, and that to when the pupil was most constricted, as well as the difference in reaction speed between the left and right eye, are measured. The status of disease is then judged based on the distance to the threshold.

All pupillary movements are recorded from the eye data obtained from the infrared cameras. The area of the pupil mentioned here is synonymous with the black pixel count. 


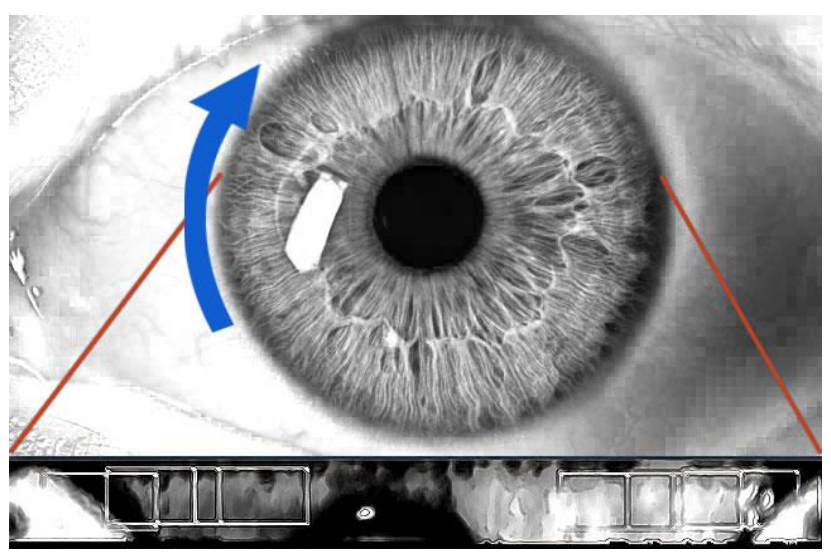

Fig. 5. Pupil analysis image.

The parameters that can be obtained using this device that we developed are as follows:

1) Initial pupil area

2) Difference in initial pupil area between left and right eyes

3) Smallest pupil area after light irradiation

4) Difference in pupil area between left and right eyes after light irradiation

5) Area of each frame

6) Number of frames to the start of constriction after light irradiation

7) Difference between left and right frames up to the start of constriction after light irradiation

8) Frame count up to when the smallest constriction is reached

9) Difference in frame count between the left and right eyes up to when the smallest is reached

10) Mydriasis rate (area/initial area of the constricted pupil)

11) Amount of change in pupil area before and after irradiation

12) Maximum miosis speed

13) Maximum miosis acceleration

The parameters obtained are presented in graph form as shown in Fig. 7. A trend in pupillary movement can be confirmed.

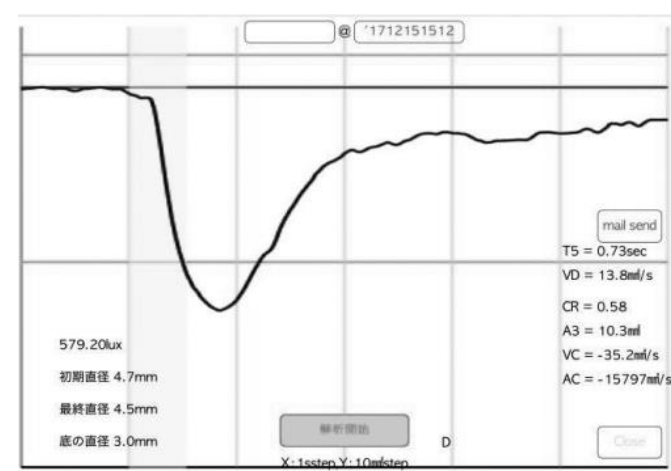

Fig. 6. Graph of analysis result (example).

\subsection{Analysis Results}

We evaluated and compared the pupillary measurements of five healthy volunteers and five patients with depression, using the pupil measuring device that we developed: first, $\mathrm{Tm}$, the time to reach the smallest pupil area from the initial pupil area; second, $T r$, the reaction time to the start of miosis; and third, 
Ts, the time to reach maximum miosis speed.

In the healthy volunteers, Tm was a mean value of approximately $1 \mathrm{sec}$, while in patients with depression it was $0.836 \mathrm{sec}$; this showed a difference. Compared with patients with depression, mean $\operatorname{Tr}$ in healthy volunteers was slightly faster, while mean Ts was shorter at approximately 0.07 sec (Table 1).

Table 1. Obtained Results of Healthy Subjects and Patients with Depression

\begin{tabular}{|c|c|c|c|c|c|c|}
\hline \multirow{2}{*}{ Subject } & \multicolumn{5}{|c|}{ Measurement result (sec) } \\
\cline { 2 - 7 } & $\begin{array}{c}\text { Healthy } \\
\boldsymbol{T} \boldsymbol{m}\end{array}$ & $\begin{array}{c}\text { Depression } \\
\boldsymbol{T} \boldsymbol{m}\end{array}$ & $\begin{array}{c}\text { Healthy } \\
\boldsymbol{T} \boldsymbol{r}\end{array}$ & $\begin{array}{c}\text { Depression } \\
\boldsymbol{T} \boldsymbol{r}\end{array}$ & $\begin{array}{c}\text { Healthy } \\
\boldsymbol{T} \boldsymbol{s}\end{array}$ & $\begin{array}{c}\text { Depression } \\
\boldsymbol{T} \boldsymbol{s}\end{array}$ \\
\hline 1 & 0.98 & 0.82 & 0.21 & 0.16 & 0.38 & 0.34 \\
\hline 2 & 1.13 & 0.81 & 0.28 & 0.19 & 0.33 & 0.32 \\
\hline 3 & 1.07 & 0.90 & 0.22 & 0.21 & 0.42 & 0.34 \\
\hline 4 & 0.87 & 0.72 & 0.20 & 0.12 & 0.35 & 0.32 \\
\hline 5 & 0.89 & 0.93 & 0.18 & 0.25 & 0.45 & 0.26 \\
\hline Average & 1.001 & 0.836 & 0.218 & 0.186 & 0.386 & 0.316 \\
\hline
\end{tabular}

Some studies have shown that when a patient has a mental or psychological disorder, the reaction towards color is that of irritation in most cases [8], [9]. This suggests that the measurements were accurately performed. A diagnosis different from that based on subjective reaction can be made from the measurement of pupil constriction by light stimulation in relation to color.

The independence of the data obtained between the groups was validated using the Probability-Density-Function, and the reliability of the analysis was determined using a ROC (receiver operating characteristic) curve. The peculiarities of pupillary movement of patients with diseases could be obtained from $T m, T r$, and $T s$. We found that $T s$ has a very high independence; when limited to the data obtained here, our device could detect the status at nearly $90 \%$ accuracy.

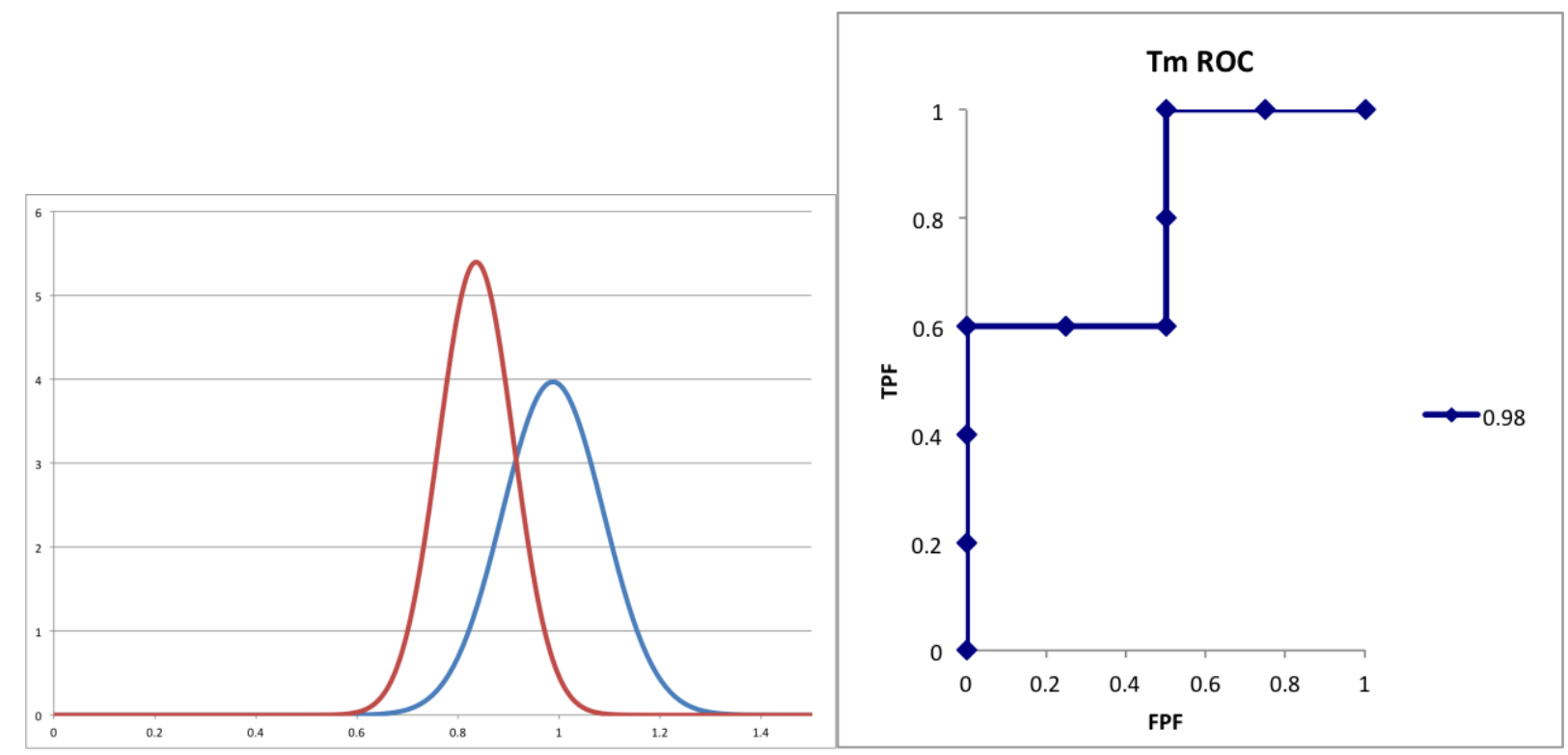

Fig. 7. The probability-density-function of Tm, the time required to reach the smallest pupil area from the initial pupil area, and the receiver operating characteristic curve (area of 0.8100). 

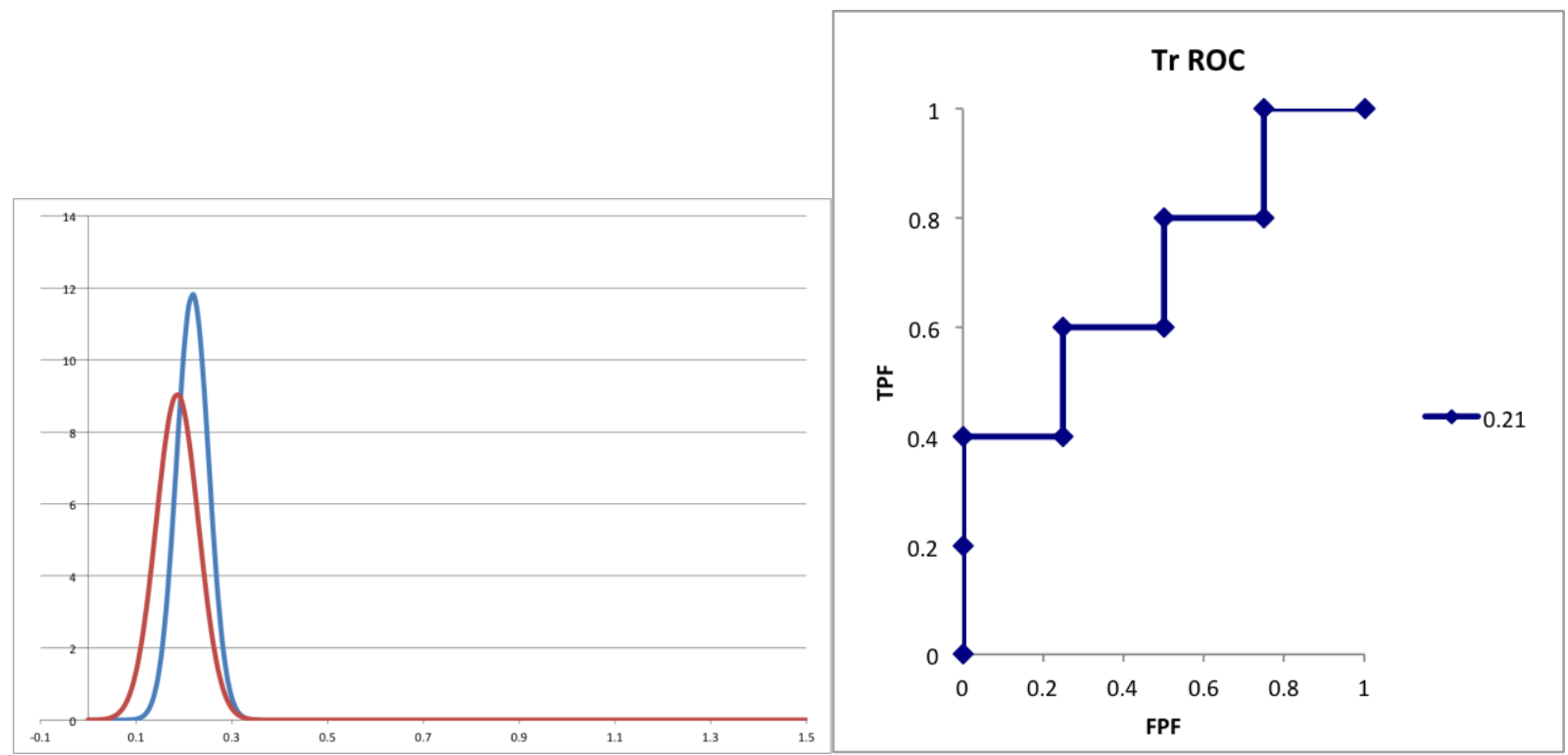

Fig. 8. The probability-density-function of $\mathrm{Tr}$, the time to the start of miosis, and the receiver operating characteristic curve (area of 0.7200).
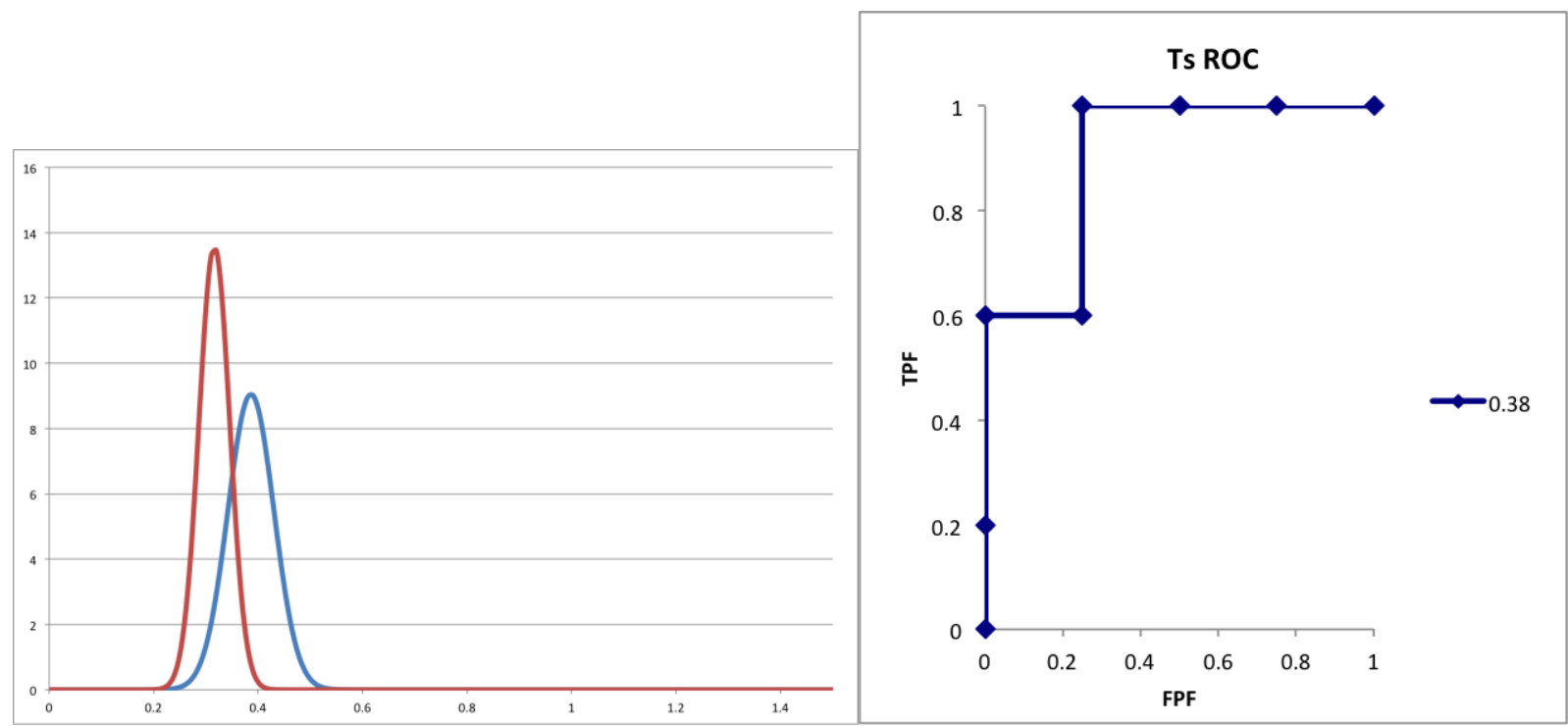

Fig. 9. The probability-density-function of Ts, the time to reach maximum miosis speed, and the receiver operating characteristic curve (area of 0.9030 ).

In this study, the pupillary size was measured in patients with depression. However, with the increasing prevalence of Alzheimer symptoms, there are patients with dull reactions towards color and light. In addition, this device may be used, even in advance diagnosis, by controlling the wavelength of light stimulation, stimulation time, and intensity.

\section{Conclusion}

In this study, we have developed a measuring device by which pupillary light reflex can be measured in real-time for the early discovery of diseases. The measurement precision is generally good, and pupillary reaction can be measured at approximately $100 \mathrm{fps}$. In the future, as a second phase of the study, we will record the pupillary movement of many healthy volunteers, as well as individuals with a history of illness, in order to determine the indices. Moreover, with regard to analysis methods, comparisons between a 
healthy volunteer group and each patient group will be made using multidimensional scaling analysis. The identification boundaries of each group will be determined, in order to establish a judgment method that will enable the early discovery of diseases. Data regarding the ocular movement of patients will be obtained using an ocular movement measurement program. We plan to cross-link ocular movement with the degree of progression of various diseases, and to perform comparative analysis between a healthy volunteer group and various patient groups.

\section{References}

[1] Haerer, A. F. (1992). DeJong's the Neurologic Examination (5th ed.). Philadelphia: Lippincott, 133-138.

[2] Posner, J. B., Saper, C. B., \& Schi, N. D. (2010). Translated and supervised by Tomio Ota. Plum and Posner's Diagnosis of Stupor and Coma (pp. 55-62). Tokyo: Medical Science International.

[3] Ritter, A. M., Muizelaar, J. P., \& Barnes, T. (1999). Brain stem blood flow, pupillary response, and outcome in patients with severe head injuries. Neurosurgery, 941-948.

[4] Litvan, I., Saposnik, G., \& Mauriño, J. (2000). Pupillary diameter assessment: Need for a graded scale. Neurology 2000, 54, 530-531.

[5] Du, R., Meeker, M., \& Bacchetti, P. (2000). Evaluation of the portable infrared pupillometer. Neurosurgery 2005, 57, 198-203.

[6] Ippei, T., Takahito, N., \& Naohiro, I. (2018). Measurement of abnormality in eye movement with autism and application for detect fatigue level. KES-IDT Intelligent Decision Technologies 2018, 187-196.

[7] Takahito, N., Ippei, T., \& Naohiro, I. (2018). Measurement of line-of-sight detection using pixel quantity variation and application for autisum. ACIS CSII 2018, 72-77.

[8] Mitsuru, K. (2016). Standard Ophthalomology, Eye Movement and Binocular Vision Function, Igaku Shoin, 234-289.

[9] Keisuke, T. (2005). Visual information processing in the blain. History of Medicine, 213(11), 1017-1024.

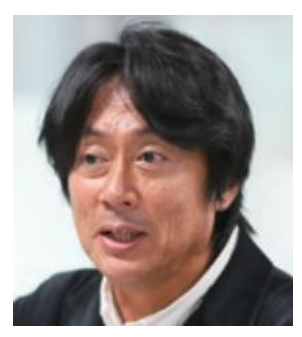

Ippei Torii received B.F.A. degree in sculpture of fine arts from Nihon University, Tokyo, Japan, in 1982. From 1982 to 1984 he was at the School of Fine Arts in Nihon University. And he received Ph.D degree in management information science from Aichi Institute of Technology in 2012. From 2015, he has been a professor in Aichi Institute of Technology. His research interests include computer graphics, image processing, biological information processing. He is a member of IEEE, ACIS and Design Research Assosiation.

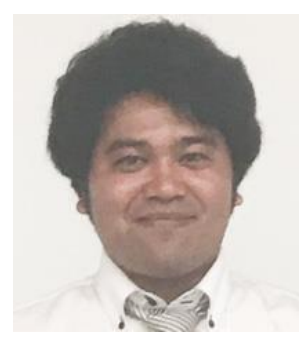

Takahito Niwa is a student belonging to the doctoral course of the Graduate School of Aichi Institute of Technology who is engaged in the laboratory of the first author. 